Supporting Information for

\title{
Band gap modulation enabled by TCNQ loading in a Ru-based metal-organic framework for enhanced NIR absorption and photothermal conversion
}

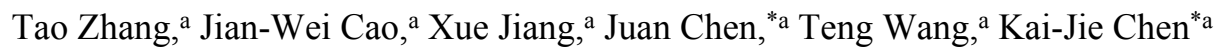

${ }^{a}$ Key Laboratory of Special Functional and Smart Polymer Materials of Ministry of Industry and Information Technology, School of Chemistry and Chemical Engineering, Northwestern Polytechnical University, Xi'an, Shaanxi, 710072

E-mail: Juan Chen: juan.chen@nwpu.edu.cn

Kai-Jie Chen : ckjiscon@nwpu.edu.cn 
General consideration. All the chemicals were purchased from commercial sources without further purification. The compound $\left[\mathbf{R} \mathbf{u}_{3}(\mathbf{b t c})_{2} \mathbf{C l}_{1.5}\right] \cdot$ guest was synthesized according to reported methods. ${ }^{1,2}$ The PXRD patterns were recorded using Rigaku D/Max-2550VB+/PC with $\mathrm{CuK} \alpha$ radiation $(\lambda=1.5418 \AA)$. The simulated PXRD patterns were calculated from the cif files downloaded from www.ccdc.cam.ac.uk/data_request/cif for free (CCDC-814515 for $\left[\mathbf{R} \mathbf{u}_{3}(\mathbf{b t c})_{2} \mathbf{C l}_{1.5}\right] \cdot$ guest, CCDC-1268182 for TCNQ solid). Room-temperature continuous-wave electron paramagnetic resonance (EPR) was performed on the Bruker ELEXSYS II equipment. Scanning electron microscope (SEM) images were recorded on SU8010 equipment, the samples were dispersed in $\mathrm{CH}_{3} \mathrm{CH}_{2} \mathrm{OH}$ ultrasonically for $30 \mathrm{~min}$. Thermogravimetric analysis (TG) was conducted on 449C Jupiter analyzer under the flowing $\mathrm{N}_{2}$. Raman spectra were recorded using Alpha300R Spectrometer equipped with a $532 \mathrm{~nm}$ TEM00 laser at Analytical \& Testing Center of Northwestern Polytechnical University. Elemental analyses (C, H and N) were recorded on an Elementar vario EL cube elemental analyzer. Gas adsorption measurements were tested by Micromeritics ASAP 2020. X-ray photoelectron spectroscopy (XPS) was conducted on a Thermo Scientific $^{\mathrm{TM}} \mathrm{K}-\mathrm{Alpha}{ }^{\mathrm{TM}+}$ spectrometer equipped with a monochromatic Al Ka X-ray source (1486.6 eV) operating at $100 \mathrm{~W}$. Samples were analyzed under vacuum $\left(\mathrm{P}<10^{-8} \mathrm{mbar}\right)$ with a pass energy of $150 \mathrm{eV}$ (survey scans) or $25 \mathrm{eV}$ (high-resolution scans). Solid-state UV-Vis-NIR spectra were tested using SHIMADZU UV-3600 UV-Vis-NIR spectrophotometer. The particle size distribution for indicated samples was recorded by LS13320 laser particle size analyzer.

Synthesis of TCNQ@[Ru $\left.\mathbf{H}_{3}(\mathbf{b t c})_{2} \mathbf{C l}_{1.5}\right]$ TCNQ@Ru-MOF. $400 \mathrm{mg}$ of $\left[\mathbf{R u}_{3}(\mathbf{b t c})_{2} \mathbf{C l}_{1.5}\right] \cdot$ guest and $100 \mathrm{mg}$ of TCNQ were mixed by grinding for $15 \mathrm{~min}$, and then the solid was charged into a matrass. The matrass was pumped to vacuum and charged with $\mathrm{N}_{2}$ for three cycles. After that, the matrass was heated at $100{ }^{\circ} \mathrm{C}$ for $12 \mathrm{~h}$ under dynamically vacuum to remove volatile guest. Subsequently, the temperature was set to $180^{\circ} \mathrm{C}$ and the valve of matrass was closed, the mixture was heated at $180^{\circ} \mathrm{C}$ for $12 \mathrm{~h}$ and then cooled down, the black powder on the bottom and the yellow solid on the wall were observed. The black solid was harvested as product and washed with $\mathrm{CH}_{2} \mathrm{Cl}_{2}$ for three times $(\sim 100 \%$, based on $\mathrm{Ru})$. CHN elemental analysis (\%) for TCNQ@Ru-MOF: found: C, $31.00 ; \mathrm{H}, 2.06$; N, 4.79, the TCNQ loading was calculated using a reported method regardless of the content of H\%. ${ }^{3}$ 0.69TCNQ@Ru-MOF was produced in exactly same procedure, only reducing reaction from 12 hours to 10 mins.

$$
\begin{gathered}
\because \quad \frac{N \%}{C \%}=\frac{14(\text { No. of } N \text { atom per guest })(\text { No. of guests per formula })}{12[\text { (No. of C atom per per MOF formula })+(\text { No. of C atom per guest })(\text { No. of guests per formula })]} \\
\because \text { No. of } N \text { atom per guest }=4 \\
\because \text { No. of C atom per guest }=12 \\
\because \text { No. of } C \text { atom per per MOF formula }=18
\end{gathered}
$$

$\therefore$ No. of guests per formula

$$
=\frac{216\left(\frac{N \%}{C \%}\right)}{14(\text { No. of } N \text { atom per guest })-12\left(\frac{N \%}{C \%}\right)(\text { No. of C atom per guest })}
$$

Photothermal property. $3.5 \mathrm{mg}$ powder samples of Ru-MOF and TCNQ@Ru-MOF were dispersed into $5 \mathrm{~mL}$ water and further ultrasonicating for $60 \mathrm{~min}$ to afford brown and black suspensions respectively. When cooling down to room temperature, $1.0 \mathrm{~mL}$ of the suspensions was taken out for photothermal measurements.

The suspension was irradiated using $980 \mathrm{~nm}$ laser sources with power of $1.262 \mathrm{~W}$ 
(LR-ISP-980/1300 mW, Changchun Lei Rui Optoelectronics Tech Co,. Ltd.) and the temperature was measured. After $16 \mathrm{~min}$, the laser was turned off and the suspension was allowed to naturally cool down and the temperature was recorded with time.

To obtain the photothermal conversion efficiency, the data should be treated with the following equations:

$$
\begin{gathered}
\eta=\frac{Q_{s}-Q_{w}}{I\left(1-10^{-A_{980}}\right)} \\
Q_{s}=h S\left(T_{\text {max }, s}-T_{\text {surr }}\right) \\
\frac{m C_{p}}{h S}=\frac{-\mathrm{d} t}{\mathrm{~d} \theta} \\
\theta=\frac{T-T_{\text {surr }}}{T_{\text {max }, S}-T_{\text {surr }}} \\
Q_{w}=h S\left(T_{\text {max }, w}-T_{\text {surr }}\right)
\end{gathered}
$$

$\eta$, photothermal conversion efficiency; $Q_{\mathrm{s}}$, photothermal heat energy generating by TCNQ@Ru-MOF; $Q_{\mathrm{w}}$, photothermal heat energy generating from back ground; $h$, heat transfer coefficient; $S$, surface area of the tube; $I$, the power of the laser; $A_{980}$, the light absorption of the suspension at $980 \mathrm{~nm} ; T_{\max , \mathrm{s}}$, the highest temperature of the suspension during the experiment; $T_{\text {surr }}$, room temperature during experiment; $T_{\max , \mathrm{w}}$, the highest temperature of water during the experiment; $C_{\mathrm{p}}$, heat capacity of water; $m$, mass of water.

Conductivity measurements. Conductivity was measured using two-probe method. The samples were pressed to pellets and silver paint was painted onto the surface of the pellet to connect wire. Then the current-voltage $(I-V)$ curves were recorded by VSP BioLogic Instruments and the conductivity was calculated by Ohm's law,

$$
\begin{aligned}
\sigma & =J / E \\
E & =V / L \\
J & =I / S
\end{aligned}
$$

where $\sigma$ is conductivity, $J$ is current density, $E$ is electric field strength, $V$ is applied voltage, $L$ is thickness of the pellet, $S$ is surface area of the pellet, $I$ is current.

DFT calculations. The non-periodic DFT calculations were performed using $\mathrm{DMol}^{3}$ module ${ }^{4}$ in Material Studio software package. The cluster model was constructed from the neutral cluster $\left[\mathrm{Ru}_{2}(\mathrm{phCOO})_{4} \mathrm{Cl}\right]$ according to a reported result. ${ }^{1}$ The spin unrestricted mode was set to deal with the cluster and the formal spin was used as initial. The van der Waals dispersion correction was applied by using TS method. ${ }^{5}$ The structures were optimized before the property calculation. The general gradient approximation (GGA) in PBE form was applied ${ }^{6}$ and the SCF tolerance was set to be $10^{-6} \mathrm{Ha}$. To plot the isosurface of HOMO (highest occupied molecular orbital) and LUMO (lowest unoccupied molecular orbital), the iso value was set to be $0.042 \mathrm{eV} / \AA^{3}$.

Table S1 Atomic coordinate in $\mathrm{Ru}_{2}\left(\mathrm{C}_{6} \mathrm{H}_{5} \mathrm{COO}\right)_{4} \mathrm{Cl}$ cluster after geometry optimization.

\begin{tabular}{|c|c|c|c|c|}
\hline Number & Atom & $\mathrm{X} / \AA$ & $\mathrm{Y} / \AA$ & $\mathrm{Z} / \AA$ \\
\hline 1 & $\mathrm{O}$ & 11.68616 & 21.81667 & 6.236963 \\
\hline 2 & $\mathrm{H}$ & 16.28205 & 20.40457 & 2.925277 \\
\hline 3 & $\mathrm{O}$ & 14.52364 & 19.65042 & 8.372761 \\
\hline 4 & $\mathrm{O}$ & 14.6378 & 21.71481 & 6.292792 \\
\hline 5 & $\mathrm{H}$ & 10.06239 & 20.57633 & 2.834737 \\
\hline
\end{tabular}




\begin{tabular}{|c|c|c|c|c|}
\hline 6 & $\mathrm{O}$ & 11.56933 & 19.75362 & 8.312848 \\
\hline 7 & $\mathrm{O}$ & 11.54717 & 18.16931 & 6.689557 \\
\hline 8 & $\mathrm{H}$ & 10.08652 & 23.62985 & 5.865005 \\
\hline 9 & $\mathrm{H}$ & 16.07743 & 19.28577 & 10.18141 \\
\hline 10 & $\mathrm{H}$ & 19.12693 & 23.64424 & 2.686081 \\
\hline 11 & $\mathrm{O}$ & 14.62103 & 20.08592 & 4.714474 \\
\hline 12 & $\mathrm{O}$ & 14.49182 & 18.0431 & 6.773322 \\
\hline 13 & $\mathrm{H}$ & 16.3304 & 23.44254 & 5.97069 \\
\hline 14 & $\mathrm{H}$ & 9.928489 & 19.40819 & 10.09908 \\
\hline 15 & $\mathrm{H}$ & 7.3143 & 23.89476 & 2.563746 \\
\hline 16 & $\mathrm{O}$ & 11.69161 & 20.2071 & 4.639745 \\
\hline 17 & $\mathrm{Ru}$ & 13.10832 & 20.78861 & 7.361089 \\
\hline 18 & $\mathrm{Ru}$ & 13.0893 & 19.11819 & 5.700536 \\
\hline 19 & $\mathrm{C}$ & 8.643009 & 23.90099 & 4.269248 \\
\hline 20 & $\mathrm{C}$ & 17.76544 & 21.9646 & 2.671413 \\
\hline 21 & $\mathrm{C}$ & 17.79399 & 23.67774 & 4.387693 \\
\hline 22 & $\mathrm{C}$ & 8.630618 & 22.17966 & 2.561418 \\
\hline 23 & $\mathrm{C}$ & 17.50756 & 17.68682 & 10.50152 \\
\hline 24 & $\mathrm{C}$ & 8.309312 & 17.97295 & 10.23101 \\
\hline 25 & $\mathrm{C}$ & 10.17984 & 22.03476 & 4.418541 \\
\hline 26 & $\mathrm{C}$ & 11.25593 & 21.31297 & 5.143051 \\
\hline 27 & $\mathrm{C}$ & 16.00374 & 17.82004 & 8.606409 \\
\hline 28 & $\mathrm{C}$ & 14.9376 & 18.54761 & 7.873995 \\
\hline 29 & $\mathrm{C}$ & 16.20045 & 21.85777 & 4.516791 \\
\hline 30 & $\mathrm{C}$ & 15.08414 & 21.17915 & 5.220136 \\
\hline 31 & $\mathrm{C}$ & 9.915729 & 18.05756 & 8.419381 \\
\hline 32 & $\mathrm{C}$ & 11.08685 & 18.69902 & 7.772521 \\
\hline 33 & $\mathrm{C}$ & 9.67023 & 23.23606 & 4.937905 \\
\hline 34 & $\mathrm{C}$ & 16.71494 & 21.32122 & 3.32528 \\
\hline 35 & $\mathrm{C}$ & 16.74282 & 23.04032 & 5.045581 \\
\hline 36 & $\mathrm{C}$ & 9.658314 & 21.51009 & 3.224928 \\
\hline 37 & $\mathrm{C}$ & 9.276386 & 16.97028 & 7.801099 \\
\hline 38 & $\mathrm{C}$ & 16.49719 & 18.35159 & 9.808909 \\
\hline 39 & $\mathrm{C}$ & 16.52891 & 16.61861 & 8.103651 \\
\hline 40 & $\mathrm{C}$ & 9.425612 & 18.55986 & 9.635681 \\
\hline 41 & $\mathrm{C}$ & 7.670418 & 16.89291 & 9.611534 \\
\hline 42 & $\mathrm{C}$ & 18.30422 & 23.14342 & 3.19904 \\
\hline 43 & $\mathrm{C}$ & 18.03093 & 16.49023 & 10.00002 \\
\hline 44 & $\mathrm{C}$ & 8.122454 & 23.37527 & 3.081379 \\
\hline 45 & $\mathrm{C}$ & 17.54063 & 15.95832 & 8.801913 \\
\hline 46 & $\mathrm{H}$ & 8.247883 & 24.83343 & 4.675239 \\
\hline 47 & $\mathrm{H}$ & 8.222528 & 21.76726 & 1.637185 \\
\hline 48 & $\mathrm{H}$ & 9.665991 & 16.59203 & 6.856307 \\
\hline
\end{tabular}




\begin{tabular}{|c|c|c|c|c|}
\hline 49 & $\mathrm{H}$ & 16.14185 & 16.21445 & 7.168341 \\
\hline 50 & $\mathrm{H}$ & 6.793698 & 16.43863 & 10.07657 \\
\hline 51 & $\mathrm{H}$ & 18.82485 & 15.97489 & 10.54345 \\
\hline 52 & $\mathrm{H}$ & 17.95113 & 15.02525 & 8.41227 \\
\hline 53 & $\mathrm{C}$ & 8.155759 & 16.39296 & 8.398283 \\
\hline 54 & $\mathrm{H}$ & 18.16389 & 21.54801 & 1.744946 \\
\hline 55 & $\mathrm{H}$ & 18.21844 & 24.59278 & 4.80332 \\
\hline 56 & $\mathrm{H}$ & 17.88408 & 18.10425 & 11.43662 \\
\hline 57 & $\mathrm{H}$ & 7.933319 & 18.36259 & 11.17812 \\
\hline 58 & $\mathrm{H}$ & 7.657404 & 15.55094 & 7.915536 \\
\hline 59 & $\mathrm{Cl}$ & 13.1314 & 22.47782 & 9.04315 \\
\hline
\end{tabular}

Table S1 Atomic coordinate in TCNQ- $\mathrm{Ru}_{2}\left(\mathrm{C}_{6} \mathrm{H}_{5} \mathrm{COO}\right)_{4} \mathrm{Cl}$ cluster after geometry optimization.

\begin{tabular}{|c|c|c|c|c|}
\hline Number & Atom & $\mathrm{X} / \AA$ & $\mathrm{Y} / \AA$ & $Z / \AA$ \\
\hline 1 & $\mathrm{O}$ & 11.51933 & 21.57831 & 5.874669 \\
\hline 2 & $\mathrm{H}$ & 16.51034 & 20.83131 & 2.871681 \\
\hline 3 & $\mathrm{O}$ & 14.28809 & 19.3545 & 8.009529 \\
\hline 4 & $\mathrm{O}$ & 14.4351 & 21.62915 & 6.182189 \\
\hline 5 & $\mathrm{H}$ & 10.33582 & 20.78749 & 2.163639 \\
\hline 6 & $\mathrm{O}$ & 11.359 & 19.30586 & 7.703981 \\
\hline 7 & $\mathrm{O}$ & 11.553 & 17.89621 & 5.943645 \\
\hline 8 & $\mathrm{H}$ & 9.786707 & 23.27538 & 5.634881 \\
\hline 9 & $\mathrm{H}$ & 15.70118 & 18.80636 & 9.945866 \\
\hline 10 & $\mathrm{H}$ & 19.10831 & 24.24859 & 3.350655 \\
\hline 11 & $\mathrm{O}$ & 14.6523 & 20.20978 & 4.432587 \\
\hline 12 & $\mathrm{O}$ & 14.48003 & 17.93639 & 6.260257 \\
\hline 13 & $\mathrm{H}$ & 15.95952 & 23.49726 & 6.210886 \\
\hline 14 & $\mathrm{H}$ & 9.652966 & 18.68773 & 9.345158 \\
\hline 15 & $\mathrm{H}$ & 7.344392 & 23.90281 & 2.129358 \\
\hline 16 & $\mathrm{O}$ & 11.73003 & 20.16829 & 4.120636 \\
\hline 17 & $\mathrm{Ru}$ & 12.89634 & 20.51475 & 7.002115 \\
\hline 18 & $\mathrm{Ru}$ & 13.10622 & 19.03331 & 5.165247 \\
\hline 19 & $\mathrm{C}$ & 8.491444 & 23.71136 & 3.951311 \\
\hline 20 & $\mathrm{C}$ & 17.88686 & 22.50228 & 2.979976 \\
\hline 21 & $\mathrm{C}$ & 17.57796 & 24.00458 & 4.859667 \\
\hline 22 & $\mathrm{C}$ & 8.804434 & 22.31244 & 1.993845 \\
\hline 23 & $\mathrm{C}$ & 17.17458 & 17.23121 & 10.17554 \\
\hline 24 & $\mathrm{C}$ & 8.205068 & 17.07146 & 9.294222 \\
\hline 25 & $\mathrm{C}$ & 10.15935 & 21.9561 & 3.969523 \\
\hline 26 & $\mathrm{C}$ & 11.20273 & 21.19228 & 4.695677 \\
\hline 27 & $\mathrm{C}$ & 15.82784 & 17.55618 & 8.189296 \\
\hline 28 & $\mathrm{C}$ & 14.80117 & 18.32572 & 7.443912 \\
\hline 29 & $\mathrm{C}$ & 16.12464 & 22.08431 & 4.589796 \\
\hline
\end{tabular}




\begin{tabular}{|c|c|c|c|c|}
\hline 30 & $\mathrm{C}$ & 15.00082 & 21.25759 & 5.093238 \\
\hline 31 & $\mathrm{C}$ & 9.870735 & 17.46598 & 7.578502 \\
\hline 32 & $\mathrm{C}$ & 10.99813 & 18.26997 & 7.044443 \\
\hline 33 & $\mathrm{C}$ & 9.501666 & 23.0164 & 4.615057 \\
\hline 34 & $\mathrm{C}$ & 16.81523 & 21.72323 & 3.420698 \\
\hline 35 & $\mathrm{C}$ & 16.50735 & 23.23087 & 5.306584 \\
\hline 36 & $\mathrm{C}$ & 9.812656 & 21.60894 & 2.653368 \\
\hline 37 & $\mathrm{C}$ & 9.39654 & 16.35488 & 6.861477 \\
\hline 38 & $\mathrm{C}$ & 16.18936 & 17.94396 & 9.491182 \\
\hline 39 & $\mathrm{C}$ & 16.45257 & 16.45263 & 7.585037 \\
\hline 40 & $\mathrm{C}$ & 9.270867 & 17.8242 & 8.799094 \\
\hline 41 & $\mathrm{C}$ & 7.731848 & 15.96632 & 8.576558 \\
\hline 42 & $\mathrm{C}$ & 18.26932 & 23.6415 & 3.697908 \\
\hline 43 & $\mathrm{C}$ & 17.79997 & 16.13522 & 9.569669 \\
\hline 44 & $\mathrm{C}$ & 8.141652 & 23.36098 & 2.642076 \\
\hline 45 & $\mathrm{C}$ & 17.43777 & 15.74622 & 8.274681 \\
\hline 46 & $\mathrm{H}$ & 7.973934 & 24.52844 & 4.456115 \\
\hline 47 & $\mathrm{H}$ & 8.531293 & 22.0413 & 0.972803 \\
\hline 48 & $\mathrm{H}$ & 9.870099 & 16.09332 & 5.914748 \\
\hline 49 & $\mathrm{H}$ & 16.15786 & 16.16391 & 6.576286 \\
\hline 50 & $\mathrm{H}$ & 6.893234 & 15.38352 & 8.963897 \\
\hline 51 & $\mathrm{H}$ & 18.57569 & 15.58459 & 10.10541 \\
\hline 52 & $\mathrm{H}$ & 17.91953 & 14.88694 & 7.805342 \\
\hline 53 & $\mathrm{C}$ & 8.328587 & 15.60861 & 7.36124 \\
\hline 54 & $\mathrm{H}$ & 18.42456 & 22.22151 & 2.072271 \\
\hline 55 & $\mathrm{H}$ & 17.87474 & 24.89297 & 5.420224 \\
\hline 56 & $\mathrm{H}$ & 17.45934 & 17.53357 & 11.18458 \\
\hline 57 & $\mathrm{H}$ & 7.741609 & 17.34768 & 10.24328 \\
\hline 58 & $\mathrm{H}$ & 7.95553 & 14.74886 & 6.801189 \\
\hline 59 & $\mathrm{Cl}$ & 12.64475 & 22.0276 & 8.852625 \\
\hline 60 & $\mathrm{C}$ & 12.6341 & 14.42465 & 1.590225 \\
\hline 61 & $\mathrm{C}$ & 12.12438 & 13.21866 & 1.193549 \\
\hline 62 & $\mathrm{C}$ & 12.61961 & 12.55136 & 0.021603 \\
\hline 63 & $\mathrm{C}$ & 13.67409 & 13.18708 & -0.71801 \\
\hline 64 & $\mathrm{C}$ & 14.19043 & 14.38872 & -0.3147 \\
\hline 65 & $\mathrm{C}$ & 13.68849 & 15.05909 & 0.851767 \\
\hline 66 & $\mathrm{C}$ & 14.22216 & 16.30379 & 1.256853 \\
\hline 67 & $\mathrm{C}$ & 12.08051 & 11.31576 & -0.39042 \\
\hline 68 & $\mathrm{C}$ & 15.27595 & 16.93667 & 0.546151 \\
\hline 69 & $\mathrm{C}$ & 13.75922 & 16.99518 & 2.397374 \\
\hline 70 & $\mathrm{C}$ & 12.52704 & 10.6635 & -1.56957 \\
\hline 71 & $\mathrm{C}$ & 11.05982 & 10.66326 & 0.348601 \\
\hline 72 & $\mathrm{~N}$ & 13.39798 & 17.59199 & 3.338946 \\
\hline
\end{tabular}




\begin{tabular}{|c|c|c|c|c|}
\hline 73 & $\mathrm{~N}$ & 16.14812 & 17.46369 & -0.03413 \\
\hline 74 & $\mathrm{~N}$ & 10.22059 & 10.13184 & 0.971165 \\
\hline 75 & $\mathrm{~N}$ & 12.89461 & 10.13889 & -2.55133 \\
\hline 76 & $\mathrm{H}$ & 12.23205 & 14.91797 & 2.476722 \\
\hline 77 & $\mathrm{H}$ & 11.3219 & 12.75039 & 1.765615 \\
\hline 78 & $\mathrm{H}$ & 14.06911 & 12.69581 & -1.60887 \\
\hline 79 & $\mathrm{H}$ & 14.996 & 14.85411 & -0.88521 \\
\hline
\end{tabular}

(a)

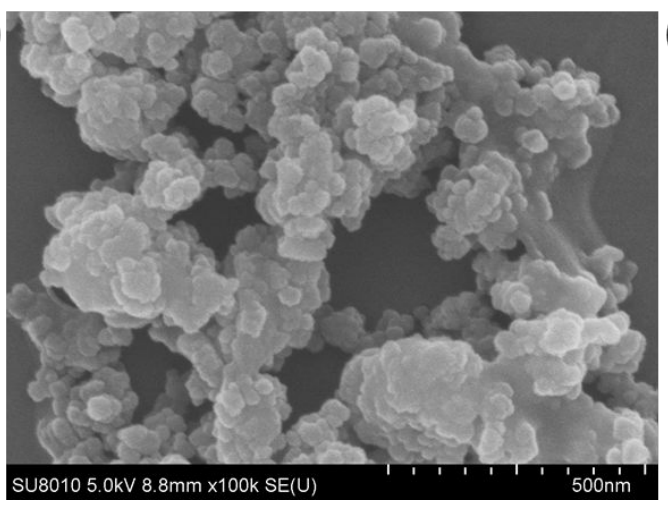

(b)

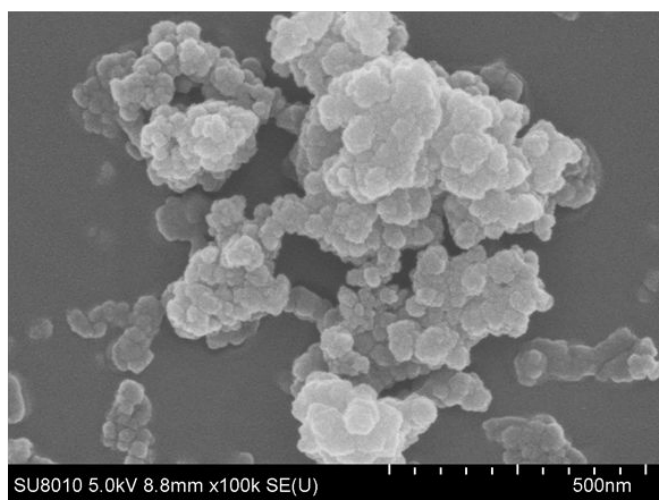

Figure S1. SEM images for (a) Ru-MOF and (b) TCNQ@Ru-MOF.

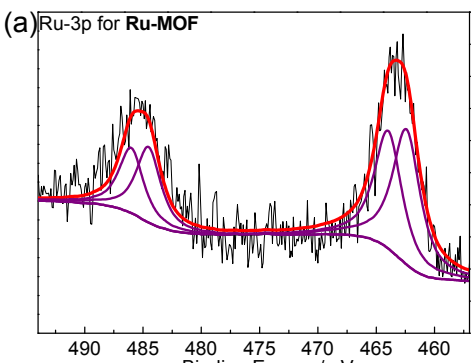

(d) Ru-3p for TCNQ@Ru-MOF

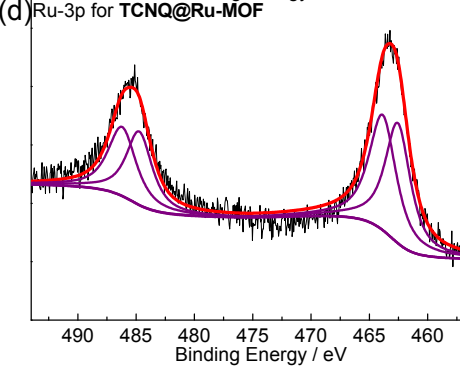

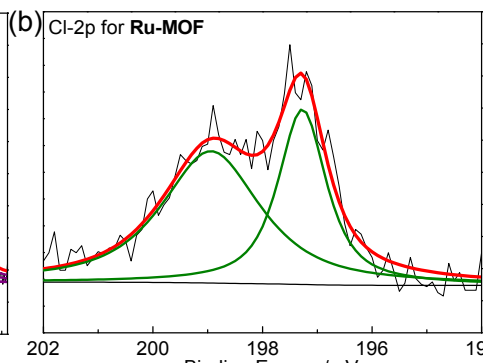

(e) Cl-2p for TCNQ@Ru-MOF

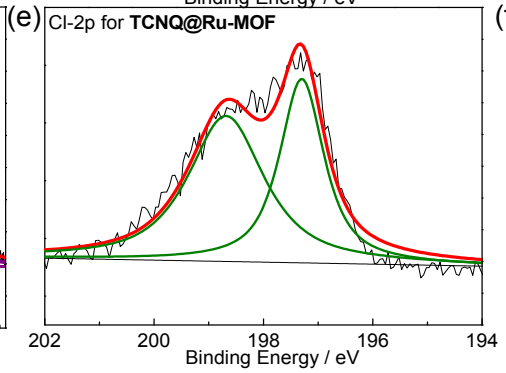

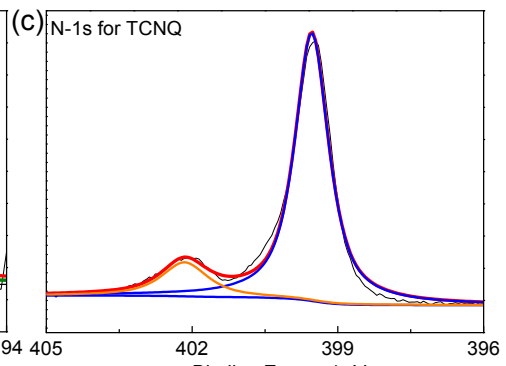

(f) $\mathrm{N}$-1s for TCNQ@Ru-MOF

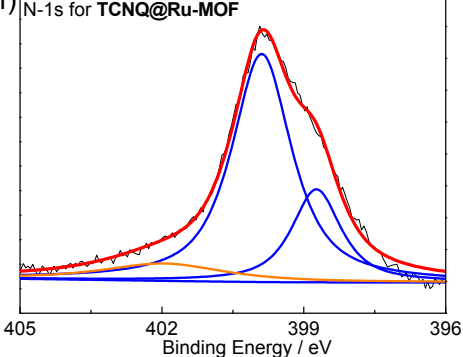

Figure S2. (a) Ru 3p XPS spectra of Ru-MOF; (b) Cl 2p XPS spectra of Ru-MOF; (c) N 1s XPS spectra for TCNQ; (d) Ru 3P XPS spectra of TCNQ@ Ru-MOF; (e) Cl 2p XPS spectra of TCNQ@Ru-MOF; (f) N 1s XPS spectra for TCNQ@Ru-MOF. 


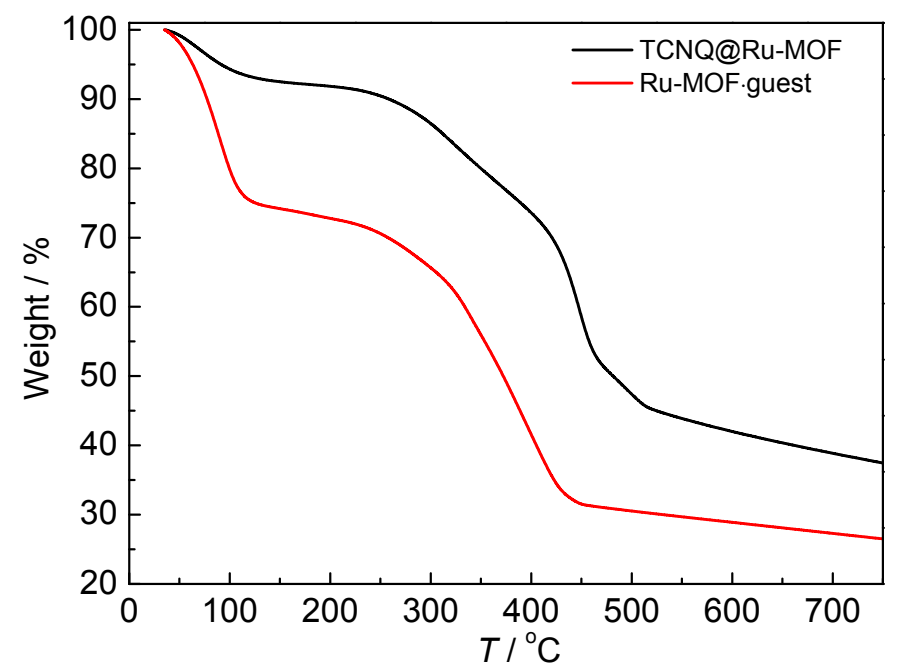

Figure S3. TG curves for indicated samples at heat rate of $10^{\circ} \mathrm{C} / \mathrm{min}$.

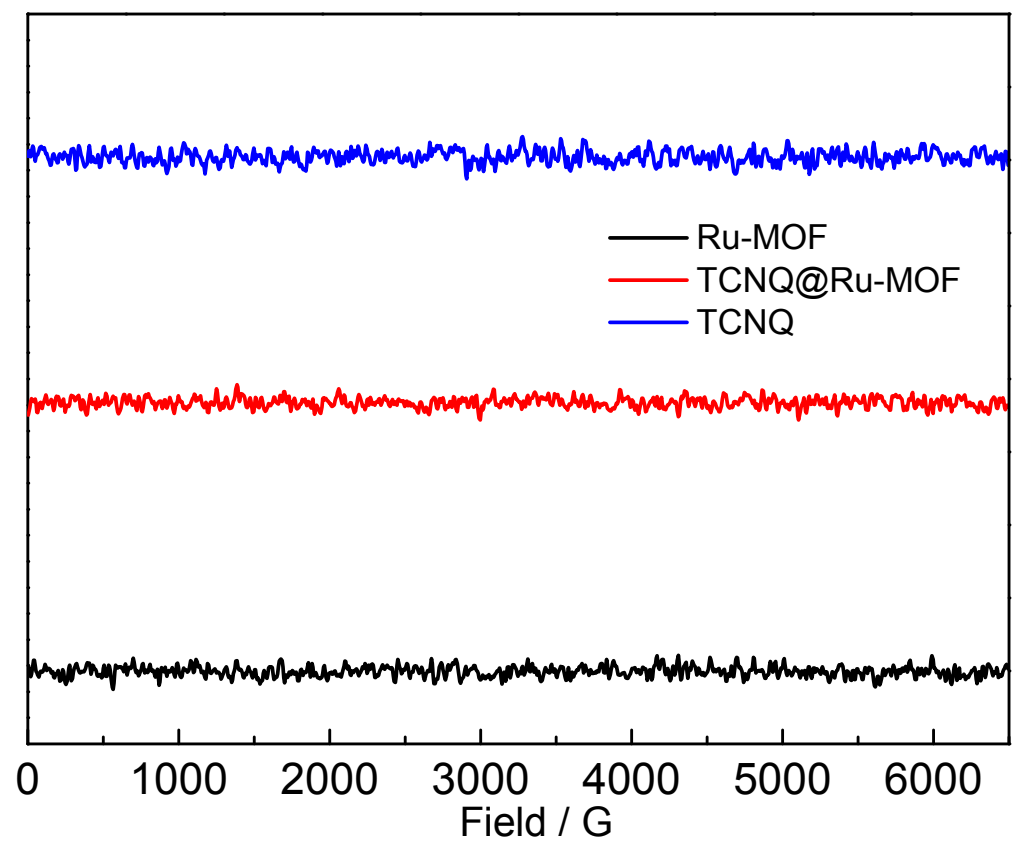

Figure S4. Room-temperature continuous-wave EPR measurements for indicated samples.

(a)

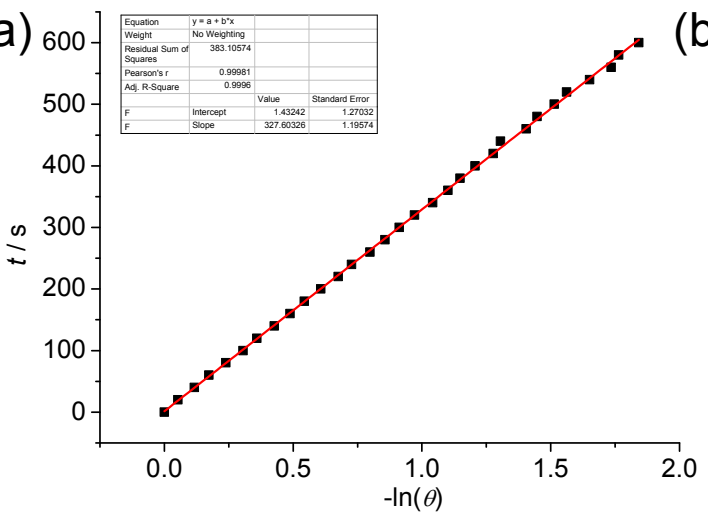

(b)

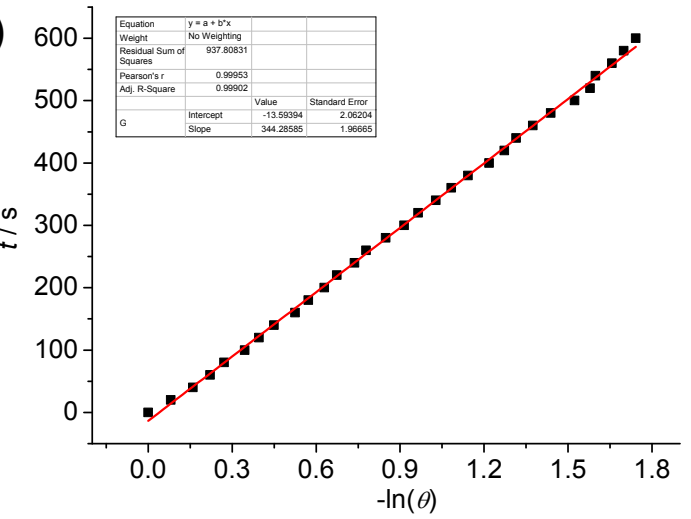

Figure S5. The $t$ vs. $-\ln (\theta)$ plots for (a) TCNQ@Ru-MOF; (b) Ru-MOF. 


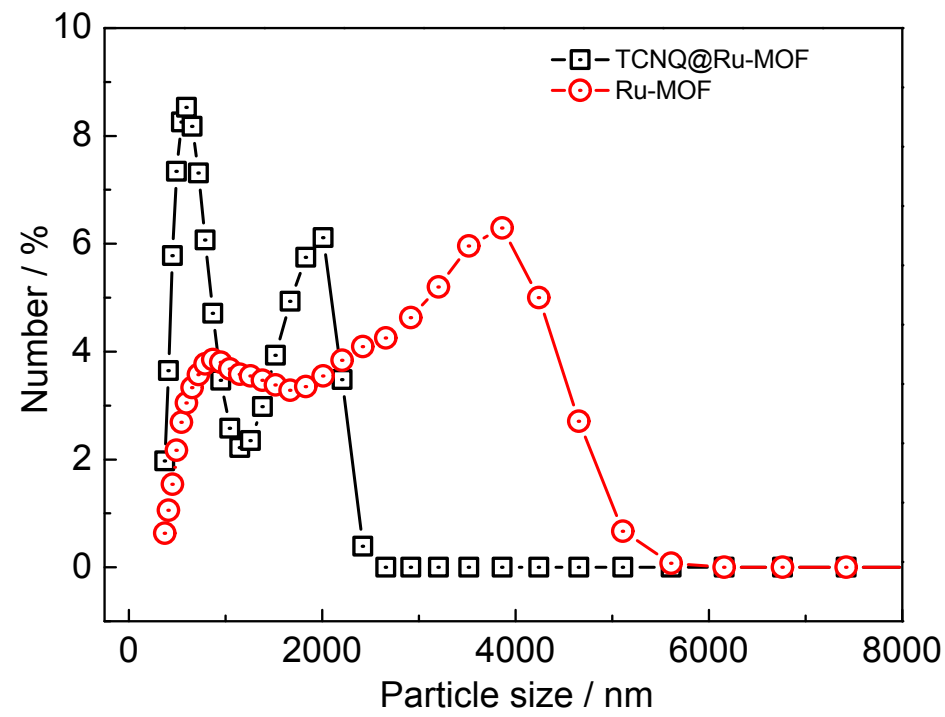

Figure S6. The particle size distribution plots of indicated samples.

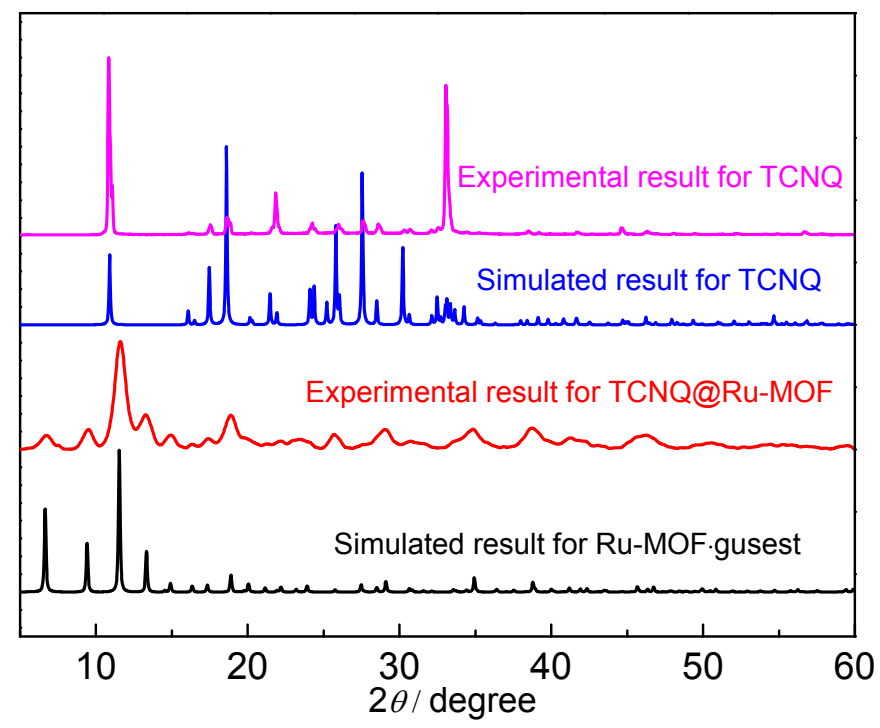

Figure S7. PXRD patterns for indicated samples. 
(a)

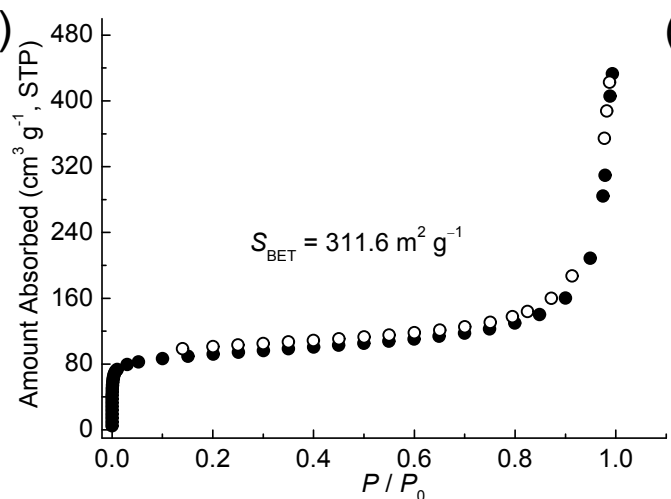

(c)

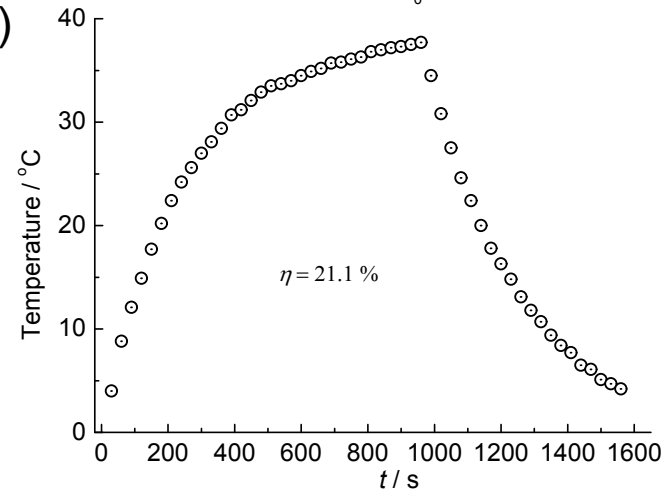

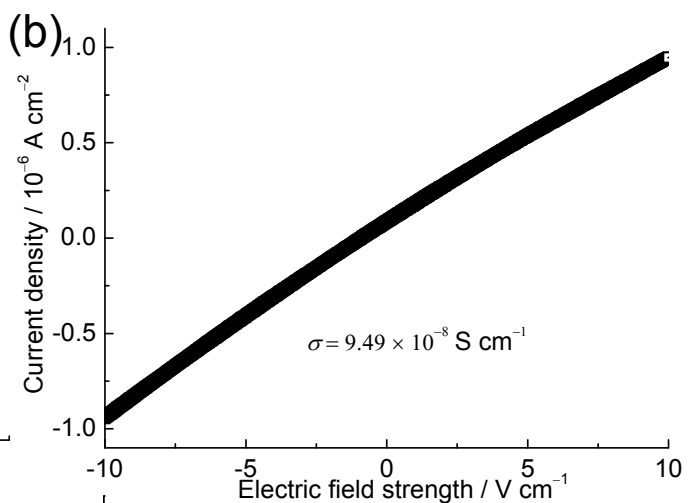

(d)

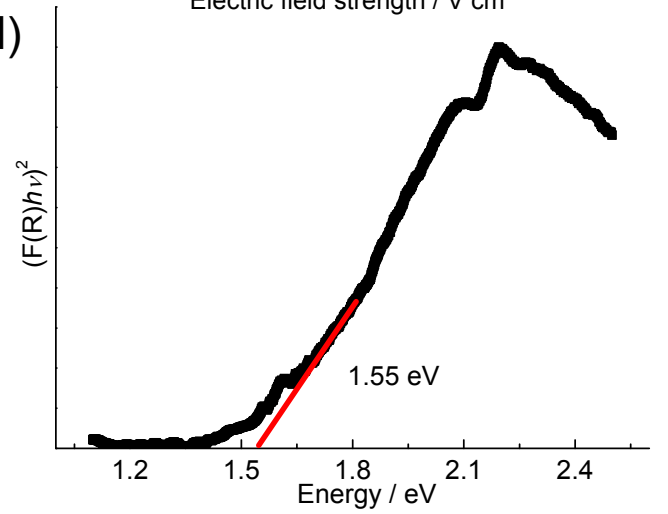

Figure S8. (a) $\mathrm{N}_{2}$ sorption data at $77 \mathrm{~K}$, (b) electrical conductivity, (c) photothermal effect and (d) UV-vis-NIR diffuse reflection spectrum of $\underline{0.69 T C N Q @ \text { Ru-MOF. }}$

\section{Reference}

1. H. Noei, O. Kozachuk, S. Amirjalayer, S. Bureekaew, M. Kauer, R. Schmid, B. Marler, M. Muhler, R. A. Fischer and Y. Wang, J. Phys. Chem. C, 2013, 117, 5658-5666.

2. O. Kozachuk, K. Yusenko, H. Noei, Y. Wang, S. Walleck, T. Glaser and R. A. Fischer, Chem. Commun., 2011, 47, 8509.

3. P. M. Usov, H. Jiang, H. Chevreau, V. K. Peterson, C. F. Leong and D. M. D Alessandro, J. Phys. Chem. C, 2017, 121, 26330-26339.

4. B. Delley, J. Chem. Phys., 2000, 113, 7756-7764.

5. A. Tkatchenko and M. Scheffler, Phys. Rev. Lett., 2009, 102, 73005.

6. J. P. Perdew, K. Burke and M. Ernzerhof, Phys. Rev. Lett., 1996, 77, 3865-3868. 